This item was submitted to Loughborough's Research Repository by the author.

Items in Figshare are protected by copyright, with all rights reserved, unless otherwise indicated.

\title{
Cost effective multiframe demosaicking for noise reduction
}

PLEASE CITE THE PUBLISHED VERSION

PUBLISHER

(C) IEEE

VERSION

VoR (Version of Record)

LICENCE

CC BY-NC-ND 4.0

REPOSITORY RECORD

Gorokhovskiy, Konstantin, James A. Flint, S. Datta, and N. Glushnev. 2019. "Cost Effective Multiframe Demosaicking for Noise Reduction”. figshare. https://hdl.handle.net/2134/6169. 
This item was submitted to Loughborough's Institutional Repository (https://dspace.lboro.ac.uk/) by the author and is made available under the following Creative Commons Licence conditions.

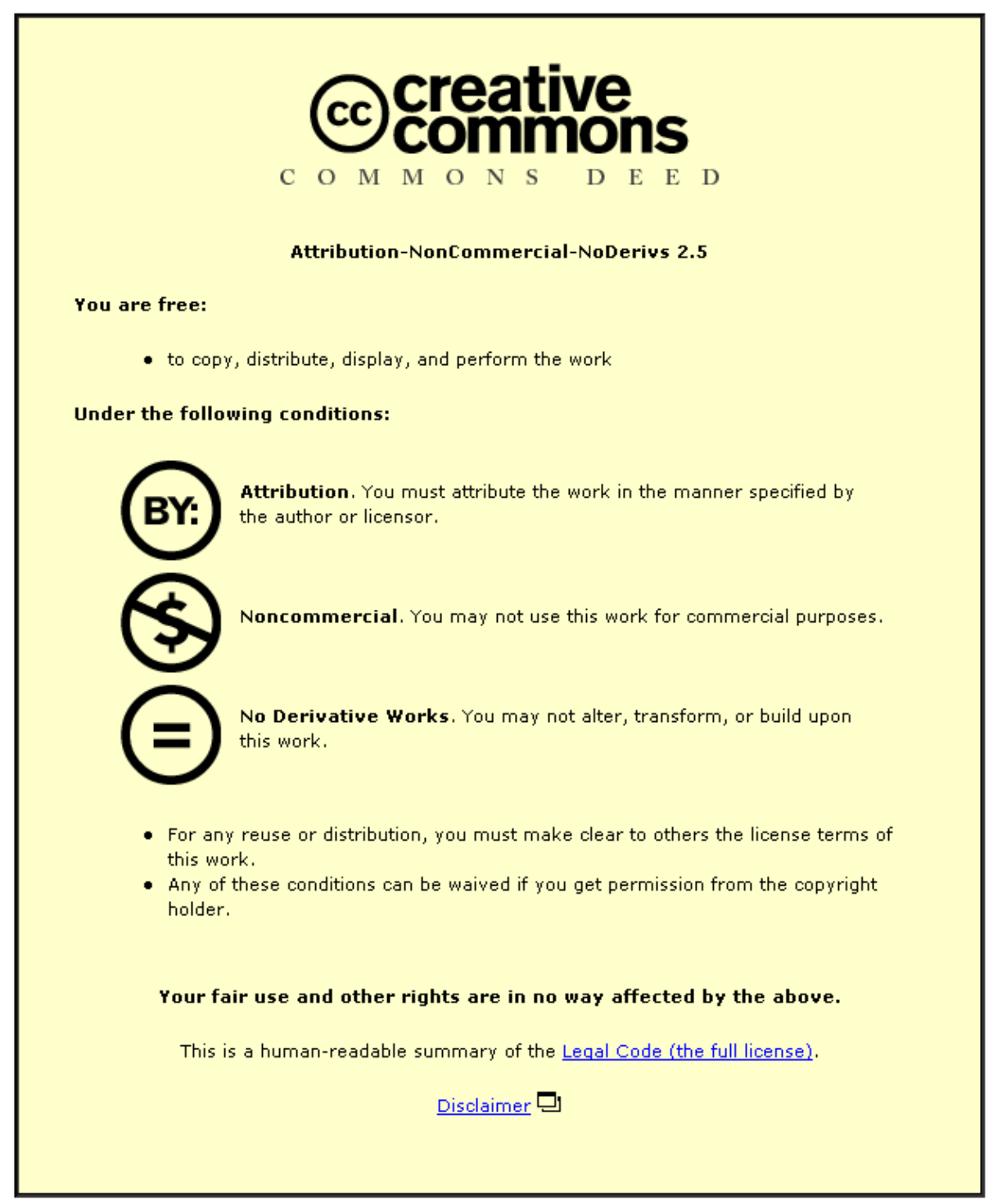

For the full text of this licence, please go to: http://creativecommons.org/licenses/by-nc-nd/2.5/ 


\title{
COST EFFECTIVE MULTIFRAME DEMOSAICKING FOR NOISE REDUCTION
}

\author{
K. Gorokhovskiy, J.A. Flint, S. Datta, and N. Glushnev
}

Department of Electronic and Electrical Engineering, Loughborough University, Loughborough, LE11 3TU, UK kgorokhovsky@apical-imaging.com, J.A.Flint@lboro.ac.uk, S.Datta@lboro.ac.uk;

Apical Limited, Loughborough Technology Centre, Epinal Way, Loughborough, LE11 3GE, UK Nikolay.Glushnev@microsoft.com

\begin{abstract}
A simple but effective multiframe demosaicking method is proposed. It is compared to a multiframe noise reduction of similar complexity. The comparison was based on computer-based simulation of a shaking camera. MSE, PSNR and NCD errors measurements were taken. Further ways of enhancing the algorithm without significant increase in complexity are proposed. The described multiframe demosaicking algorithm is suitable for mass production devices such as mobile phones of digital cameras. Its primary goal is to replace more expensive mechanical motion compensation systems.
\end{abstract}

Index Terms - multiframe noise reduction, demosaicing, demosaicking, temporal noise reduction, multiframe demosaicking

\section{INTRODUCTION}

Digital cameras and so-called camera-phones are now widely spread. Although, image quality from them has improved drastically in recent years, still, it is not comparable to human vision capabilities especially under low light conditions. One of the main problems is noise. Current cameras perform on their physical limits and photon noise is dominant. On physical level, this type of noise can be reduced by increasing number of photons detected by each cell on a sensor. Usually, the solutions are: increasing of optical efficiency of a lens system or increasing exposure times. Improving optical efficiency is expensive as the complexity of the lens grows disproportionately relative to its quality, not to mention that camera often needs to be small. Longer exposures, in turn, produce motion blur

The authors gratefully acknowledge the funding of Apical Limited, UK who supported this work.

Apical Limited, UK < www.apical-imaging.com> is a world-wide leader in embedded image enhancement technologies for variety of electronic devices including digital cameras and mobile phones. (contact: info@apical-imaging.com)

Nikolay Glushnev was with Apical Limited, UK. He is now a Software Design Engineer at Microsoft Corp., Redmond, WA 98052 USA (e-mail: Nikolay.Glushnev@microsoft.com).

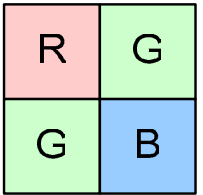

Fig. 1. Bayer pattern. There are variations of it but main idea remains - two green samples per one red and blue. This pattern is chosen primarily because green colour represents luminosity.

which can be compensated mechanically or electronically. Taking into account the generally falling cost of electronic components electronic motion compensation becomes more and more attractive in terms of quality per unit cost.

Both frame-based demosaicking and multiframe noise reduction are well developed areas on their own. The combination of these two methods only recently received a proper attention [1]. However, there is still lack of simple but effective methods which can be implemented in existing devices.

In this work the proposed method of multiframe demosaicking is compared to combination of simple framebased demosaicking (see Figure 1) and multi-frame noise reduction. The comparison is carried out using computerbased simulation of a series of shots which are shifted and rotated, then mosaicked. After that, Poisson noise is added to simulate the photon noise of a photo sensor.

The original prerequisite for the proposed algorithm is that it can be put into a camera image processing pipeline without a significant increase in cost. This leads to the following requirements:

(a) The method should not consume too much memory (not more than 4 image frames) even if the technique involves merging many more frames.

(b) It should be online or, in other words, user should receive the result just after the shot (no time-consuming post processing allowed).

It is clear from the requirements that algorithm should be stream based and data should be accumulated and processed "on the fly".

Having many images of the same scene it is possible to use wide variety of super-resolution algorithms ([2]- [5]). The requirements for memory and computational power do 


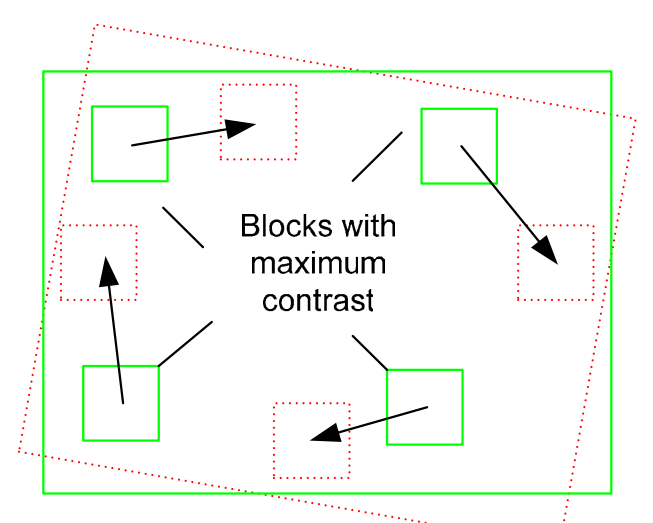

Fig. 2. Global motion estimation using block matching. We can assume that if rotation is small motion blocks are just shifting without rotation

not allow an increase in the resolution except by reducing noise.

\section{DESCRIPTION OF METHODS}

There were three independent modules implemented:

- Global motion estimation

- Simple multiframe noise reduction based on "affine transform and add" method

- Multiframe demosaicking based on "affine transform and weighted add" method

Using the same motion estimation for both methods it is possible to exclude it from the comparison. The description of used global motion estimation is available below but its optimization is a separate subject and is not described here.

\subsection{Global Motion Estimation}

As the global motion estimation was not an essential part of the comparison the simplest exhaustive search was taken as a basis. It was assumed that global motion of the frame can be described as affine transform with relatively small coefficients so that for small area of image it is just shift in two dimensions. Formulas (1) and (2) define this condition:

$$
\left[\begin{array}{c}
x^{\prime} \\
y^{\prime} \\
1
\end{array}\right]=\left[\begin{array}{ccc}
a_{11} & a_{12} & a_{13} \\
a_{21} & a_{22} & a_{23} \\
0 & 0 & 1
\end{array}\right] \cdot\left[\begin{array}{c}
x \\
y \\
1
\end{array}\right]
$$

Where

$$
a_{21}<<1, a_{12}<<1
$$

Then a limited number of blocks with maximal contrast were selected. The target is to find shifts in these blocks and to calculate the global motion using linear regression or robust fitting [6]. Figure 2 illustrates this.

Exhaustive search block matching algorithm was taken from [7]. It was modified to introduce a penalty term for big
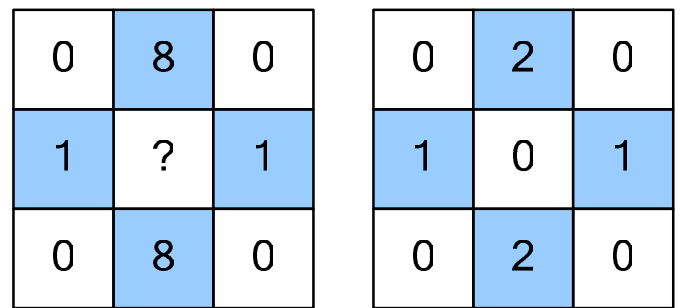

Fig. 3. What is expected value of blue color in central location (indicated with a question mark)?

motion vectors. Having two vectors with equal cost the shortest will be selected.

\subsection{Noise reduction}

There were the steps taken for noise reduction:

1. Demosaic every given frame with bilinear or more sophisticated demosaicking method.

2. Find global motions between key frame (in our case simply first frame from the sequence) and the rest of frames using demosaicked images.

3. Apply backward affine transform to match with key frame.

4. Take average colours from matched frames for each pixel.

The following methods were also used instead of bilinear demosaicking

1. Variable Number of Gradients [9]

2. High quality linear interpolation [10]

3. Hue preserving interpolation [11]

It is important to note that the above described scheme is memory efficient as images can be processed one by one, or in other words, amount of memory required does not depend on number of frames in one sequence.

\subsection{Multiframe demosaicking}

The multiframe demosaicking consists of the following steps:

1. Initial demosaicking. Bilinear demosaicking is used here

2. Find the global motion between frames based on the reconstructed images from the first step

3. Apply a backward transform to the Bayer pattern and Bayer pattern mask

Fusing Bayer frames and masks using an averaging method is described below.

Steps 1 and 2 are the same as for noise reduction, but step 3 requires a more detailed explanation.

Let us assume that value of one colour in pixel with coordinates $\left(x_{1}, y_{1}\right)$ is

$$
s_{1}=s\left(x_{1}, y_{1}\right)
$$


It is possible to describe a probability density function for a pixel with coordinates $\left(x_{1}, y_{1}\right)$ with known neighbour in point $\left(x_{2}, y_{2}\right)$ as follows

$$
P\left(s_{1} \mid s_{2}, \rho\left(s_{1}, s_{2}\right)\right)=P\left(s_{2} \mid s_{1}, \rho\left(s_{1}, s_{2}\right)\right)
$$

Where $\rho\left(s_{1}, s_{2}\right)$ is the distance between two coordinates of pixels. It is also assumed that both values $s_{1}$ and $s_{2}$ are equally likely. Equation (3) is a simplified Bayes rule. For $\mathrm{N}$ neighbouring pixels we have

$$
\begin{aligned}
P(s)=\frac{1}{c} \sum_{i=1}^{N} P\left(s_{i}\right) \cdot P\left(s \mid s_{i}\right) & = \\
& =\frac{1}{c} \sum_{i=1}^{N} P\left(s_{i}\right) \cdot f\left(\rho\left(s, s_{i}\right)\right)
\end{aligned}
$$

Where $P\left(s_{i}\right)$ is a distribution of individual colour and $c$ is a normalization coefficient. Form of $P\left(s \mid s_{i}\right)=f\left(\rho_{i}\right)$ can be estimated from experimental data for a given set of images. The distribution should look close to Gaussian with centre in $\rho=0$. Mathematical expectation of $s$ equals:

$$
\langle s\rangle=\frac{1}{c} \sum_{i=1}^{N}\left\langle s_{i}\right\rangle \cdot f\left(\rho_{i}\right)
$$

Where normalization coefficient:

$$
c=\sum_{i=1}^{N} f\left(\rho_{i}\right)
$$

In practice the described formula can be calculated using cumulative value and weight masks for every colour plane.

As we are working with discrete images to use formula (5) we only need to know the cumulative sum of all the values and the number of values dropped into a given location. An example is shown in the Fig. 3. For the given distribution of pixels, Equation (5) will give us value of 3 . The distance from the four neighbouring pixels is the same

TABLE I

RESULTS OF MULTIFRAME DEMOSAICKING COMPARING TO TEMPORAL NOISE REDUCTION ON KODAK IMAGE SET

\begin{tabular}{c|c|c|c|c|c|c}
\hline \hline \multirow{2}{*}{$\begin{array}{c}\text { Number } \\
\text { of frames in } \\
\text { a set }\end{array}$} & \multicolumn{2}{|c|}{ Temporal Noise Reduction } & \multicolumn{2}{c}{ Multiframe Demosaicking } \\
\cline { 2 - 7 } & MSE & PSNR & NCD & MSE & PSNR & NCD \\
\hline 5 & 0.001408 & 29.32 & 0.07157 & 0.001012 & 30.75 & 0.08374 \\
10 & 0.001373 & 29.45 & 0.06148 & 0.000854 & 31.43 & 0.06700 \\
15 & 0.001385 & 29.40 & 0.05876 & 0.000836 & 31.54 & 0.06172 \\
20 & 0.001367 & 29.48 & 0.05602 & 0.000779 & 31.92 & 0.05572 \\
25 & 0.001340 & 29.57 & 0.05424 & 0.000750 & 32.08 & 0.05290 \\
30 & 0.001360 & 29.54 & 0.05354 & 0.000763 & 32.14 & 0.05102 \\
35 & 0.001358 & 29.53 & 0.05272 & 0.000755 & 32.12 & 0.04917 \\
40 & 0.001341 & 29.58 & 0.05186 & 0.000744 & 32.19 & 0.04767
\end{tabular}

Proc. of the 2007 15 th Intl. Conf. on Digital Signal Processing (DSP 2007) and hence $P\left(s \mid s_{i}\right)$ are equal and can be cancelled.

\section{COMPARISON OF METHODS}

The methods were compared using raw images generated from "Kodak Image Set". Images were downscaled to reduce simulation time. The aim was to simulate the image sequence from the real camera. Simulation was introduced into the comparison because with real raw images it is impossible to get precise colours for every pixel.

The following assumptions are used when comparing the two methods:

1. Overall exposure time of a set of shots is short (less than $1 / 4$ second)

2. More than 16 frames to capture (each less than $1 / 64$ second)

3. There is only rotation and shift taking place (no scaling)

4. Rotation is no more than 5 degrees between any two individual images in a set.

5. Motion shift is no more than 100 pixels between any two images in a set. This restriction is applied only for faster simulation.

For error measurement MSE, PSNR, NCD formulae were taken. MSE and PSNR are

$$
\begin{aligned}
& \sigma_{M S E}= \\
& =\frac{1}{W \cdot H \cdot C} \sum_{y=1}^{W} \sum_{x=1}^{H} \sum_{c=1}^{3}\|O(x, y, c)-R(x, y, c)\|^{2} \\
& \sigma_{P S N R}=10 \cdot \log _{10}\left(\frac{1}{\sigma_{M S E}}\right)
\end{aligned}
$$

Here we assume that colour values are within the range $[0,1]$.

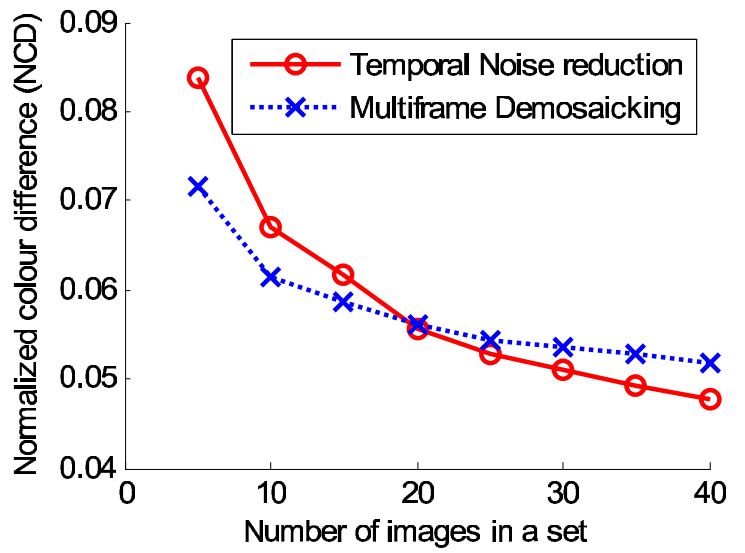

Fig. 4. Dependence of normalized color difference from number of images available for warping. As can be seen multiframe demosaicking becomes more effective with number of images higher than 35 . 


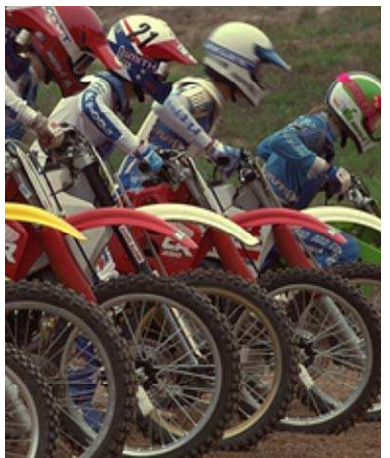

(a)

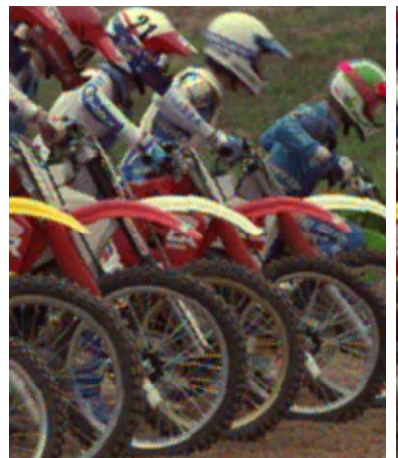

(b)

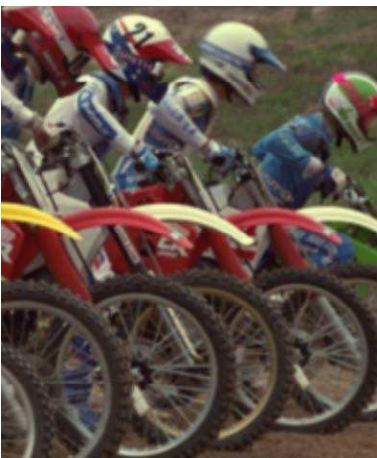

(c)

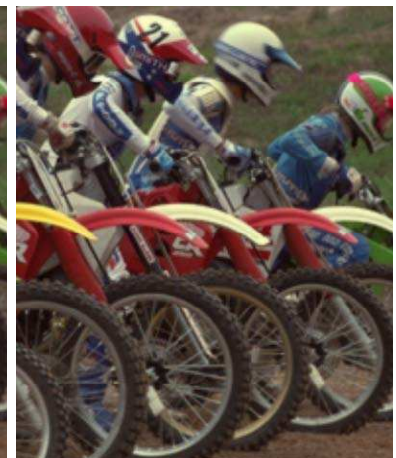

(d)

Fig. 5. From left to right: (a) original image, (b) one noisy image from the set of 32, (c) result of temporal noise reduction on 32 images, (d) result of multiframe noise reduction on 32 images.

NCD stands for Normalized Colour Difference and is used to quantify the perceptual colour difference and is defined as follows:

$$
\sigma_{N C D}=\frac{\sum_{x, y} \sqrt{\left(L_{o}-L_{r}\right)^{2}+\left(u_{o}-u_{r}\right)^{2}+\left(v_{o}-v_{r}\right)^{2}}}{\sum_{x, y} \sqrt{L_{o}^{2}+u_{o}^{2}+v_{o}^{2}}}
$$

Where $L, U, V$ are lightness and chrominance components of result and original images in CIELUV colour space. They are converted from sRGB colour space through CIEXYZ colour space. The conversion sequence was the following:

$$
s R G B \rightarrow \text { linear } R G B \rightarrow C I E X Y Z \rightarrow C I E L U V
$$

\section{RESULTS}

The simulation was performed for different number of images in a set varying from 5 to 40 with a step size of 5 . The results are shown in the Table I. As can also be seen from the graph in Fig. 4 the multiframe demosaicking becomes more effective compared to temporal noise reduction as the number of images in one set increases. Actual image samples from processing are shown in Fig. 5.

It is important to note that the proposed method is not based on the specific structure of classic Bayer filter layout and can be easily adapted for alternative filter patterns such as described in [12].

\section{CONCLUSION}

The proposed method of multiframe demosaicking showed clear advantage over temporal noise reduction on large image sets (more than 35 images). It is also simple to implement in hardware of modern digital camera or mobile phone. To get better results with a small number of images in a set multiframe demosaicking can be improved in an

adaptive way such as spatial filtering kernel for uniform surfaces and temporal filtering for edges.

\section{REFERENCES}

[1] S. Farsiu, M. Elad, and P. Milanfar,"Multiframe Demosaicking and Super-Resolution of Color Images", IEEE Trans. Image Process., vol. 15, no. 1, pp. 141-159, Jan. 2006.

[2] M. Irani and S. Peleg, "Improving resolution by image registration," CVGIP: Graph. Models Image Process., vol. 53, pp. 231-239, 1991.

[3] S. Peleg, D. Keren, and L. Schweitzer, "Improving image resolution using subpixel motion," CVGIP: Graph. Models Image Process., vol.54, pp. 181-186, Mar. 1992.

[4] M. Elad and A. Feuer, "Restoration of single super-resolution image from several blurred, noisy and down-sampled measured images," IEEE Trans. Image Process., vol. 6, no. 12, pp. 1646-1658, Dec. 1997.

[5] A. Zomet and S. Peleg, "Efficient super-resolution and applications to mosaics," in Proc. Int. Conf. Pattern Recognition, Sep. 2000, pp. 579-583.

[6] Holland, P.W., and R.E. Welsch, "Robust Regression Using Iteratively Reweighted Least-Squares, " Communications in Statistics: Theory and Methods, 1977, A6, 813-827.

[7] Aroh Barjatya, "Matching Algorithms for Motion Estimation, " 2004, $<$ http://www.mathworks.com/matlabcentral/fileexchange/load File.do?objectId $=8761>$

[8] Chang, Ed. et.al., "Color Filter Array Recovery Using a Threshold-based Variable Number of Gradients", Proceedings of SPIE, Jan 1999

[9] Henrique Malvar, Li-wei He, and Ross Cutler, "High-quality linear interpolation for demosaicking of Bayer-patterned color images. IEEE International Conference on Speech, Acoustics, and Signal Processing," 2004.

[10] D. R. Cok, "Signal processing method and apparatus for producing interpolated chrominance value in a sampled color image signal," U.S. Patent No. 4,642,678, 1987.

[11] 24 scanned images, "Estman Kodak (c) photographic color image database", 1993.

[12] K. Gorokhovskiy, J.A. Flint, S. Datta, "Alternative color filter array layouts for digital photography", Research in Microelectronics and Electronics 2006, Ph. D., June 2006 